\title{
Numerical study of fuel and turbulence distributions in an automotive-sized scavenged pre-chamber
}

This article presents a numerical study of the fuel and turbulence distributions in a pre-chamber at spark-time. The study has been conducted in the framework of the H2020 Gas-On project, dealing with the development of a lean-burn concept for an automotive-sized gas engine equipped with a scavenged pre-chamber. The test case considered studies a 7-hole pre-chamber with circumferentially-tilted orifices mounted on the cylinder head of a rapid compression-expansion machine (RCEM), consistent with the experimental test rig installed at ETH Zurich. An accurate description of turbulence and fuel distributions are key quantities determining the early flame development within the pre-chamber. Both quantities have an influence on the overall combustion characteristics and therefore on the engine performance. For this purpose, computational fluid dynamics (CFD) is employed to complement experimental investigations in terms of data completeness. The performance of the Reynolds-averaged Navier-Stokes (RANS)-based turbulence model is compared with large-eddy simulation (LES) through ensemble averaging of multiple LES realizations, in which the fuel injection rate evolution into the pre-chamber has been perturbed. Overall, RANS results show that the distributions of the turbulent kinetic energy and fuel concentration at spark-time agree well with the LES ensemble-averaged counterparts. This constitutes a prerequisite in view of the combustion phase and the accuracy reported provides further confidence in this regard.

Key words: scavenged pre-chamber, RANS, LES, mixture formation

\section{Introduction}

Efforts to reduce $\mathrm{CO}_{2}$ emissions from internal combustion engines have led to the use of natural gas as a fuel in lean-burn spark ignition engines. A widely used technology in lean-burn gas engines is pre-chamber ignition systems, in which the external ignition source is located in a separate small volume, connected to the main chamber via small orifices [6, 17]. This setup allows the design for favorable ignition conditions near the ignition source, which result in fast and repeatable early flame propagation. The use of prechamber systems in engines allows the combustion of very lean/diluted mixtures, resulting in higher efficiencies and lower $\mathrm{NO}_{\mathrm{x}}$ emissions.

Research in the field of pre-chamber combustion has been extensive in the past years, aiming to increase our understanding and allow the practical application of such systems.

Computational fluid dynamics (CFD) of pre-chamber combustion system has been increasingly employed in the last decade to complement experimental testing. Most of the work has been focused on RANS models (e.g. [2, 5, 8, $10,16,21-23])$ and some LES studies start to appear (e.g. $[1,4,7,18,19])$. A large variety of pre-chamber geometries have been considered, from stylized shapes with singleorifice nozzles up to close-to-production complex designs with multiple orifices. It is well known that the spatial distribution of the fuel concentration at the time of sparking plays a pivotal role on the flame development within the pre-chamber in gas engines equipped with a scavenged prechamber. The combustion within the pre-chamber is in turn a determining factor for the behaviour of the turbulent jets exiting the pre-chamber and therefore this influences the combustion of the premixed charge in the main chamber.

A detailed assessment of the turbulence and fuel distributions within the pre-chamber is a very challenging task; the main reason for this is twofold: First, spatial fuel distribution in real-geometry pre-chamber cannot be directly measured. Second, results of CFD simulation using a RANS approach for such a complex flow depend considerably on the choice of the turbulence model parameters, and results cannot be verified through experimental data. The approach employed in this paper is based on a socalled "numerical experiment", where simulation results from LES and RANS are compared in a consistent manner, in order to assess the RANS model performance.

The analysis is focused on the turbulence and fuel distributions at the time of ignition, in order to evaluate the RANS model performance in comparison to the LES, in view of the reactive phase.

The investigated pre-chamber shape was developed using CFD analysis with VECTIS CFD package within Horizon 2020 GasOn project. The project focusses on the reduction of emissions in natural gas passenger vehicle applications using scavenged pre-chamber technology to facilitate lean operation at high compression ratios.

Cold flow simulations have been selected as the focus of this publication in order to illustrate the approach to the verification of simulations based on a purely numerical study. Further development, application and validation of the computational methodology developed within the Horizon 2020 GasOn project to complex reacting flow simulations in pre-chamber and spark ignited engines is described in detail in $[3,9,11,13-15]$.

The remainder of the paper is structured as follows. The methodology section presents the rapid compressionexpansion machine and pre-chamber characteristics, the LES and RANS setups, as well as the fuel injection strategy. In the results section, the results of the RANS turbulence model are compared to multi-cycle averaged LES in 
terms of fuel and turbulence distribution within the prechamber. The paper closes with conclusions.

\section{Methodology}

\subsection{Rapid Compression-Expansion Machine (RCEM)}

The current study was performed in a Rapid Compression Expansion Machine (RCEM). The RCEM operates in a single cycle mode (compression-expansion) and combines excellent optical access with high flexibility in independently changing parameters, such as mixture composition, start of ignition, initial chamber conditions, etc. The RCEM employs a freely floating piston with an electrically controlled and pneumatically and hydraulically actuated driving system. A schematic of the RCEM, showing the most important components, is shown in Fig. 1. The most important technical characteristics are summarized in Table 1 , while further details can be found in [14].

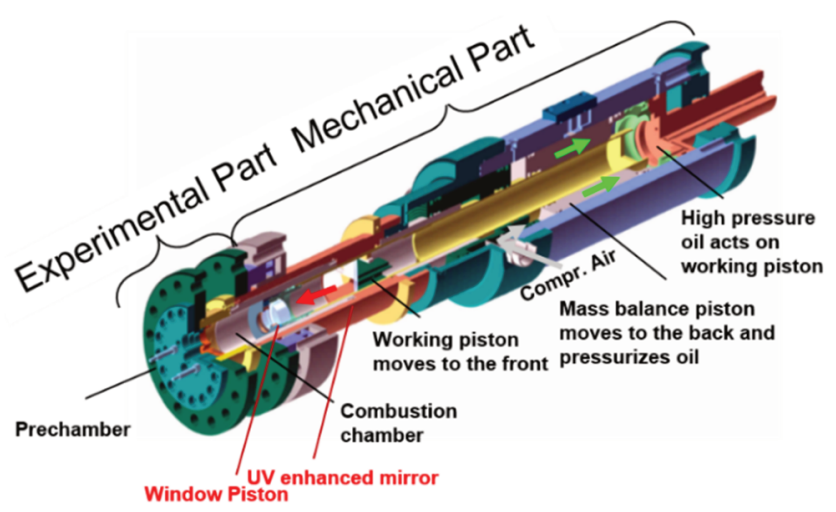

Fig. 1. Drawing of the RCEM, showing the most important components

The RCEM bore diameter is $\mathrm{B}=84 \mathrm{~mm}$ and the quartz piston has a top hat bowl shape with diameter of $52 \mathrm{~mm}$ and depth of $2.2 \mathrm{~mm}$. The piston stroke is adjustable ( $\mathrm{s}=$ $=120-250 \mathrm{~mm}$ ), and for this study was set at maximum. The temperature of the cylinder head and liner was set to $383 \mathrm{~K}$ using different heating elements and measured by 6 K-type thermocouples. Differently than in an engine, the filling process of the RCEM cylinder is occurring well before the compression and thus no turbulence exists in the main chamber at the beginning of the stroke.
The pre-chamber used is a prototype, and was positioned centrally and in a plane normal to the cylinder axis, similarly to its placement in an engine cylinder. The prechamber has 7 nozzles of $1.5 \mathrm{~mm}$ in diameter, which are placed at an angle to the cylinder axis in order to induce a swirling flow within the pre-chamber during compression.

Table 1. Technical characteristics of the Rapid Compression Expansion Machine

\begin{tabular}{|l|c|}
\hline \multicolumn{1}{|c|}{ Parameter } & Description \\
\hline Bore $(\mathrm{B})$ & $84 \mathrm{~mm}$ \\
\hline Stroke length $(\mathrm{s})$ & $\begin{array}{c}\text { Adjustable from } 120-249 \mathrm{~mm} \\
\text { (here } 249 \mathrm{~mm})\end{array}$ \\
\hline Compression stroke $(\varepsilon)$ & $5-30$ \\
\hline Loading pressure $\left(\mathrm{P}_{\text {load }}\right)$ & $1-3$ bar (here 1.2 bar) \\
\hline Max. cylinder pressure $\left(\mathrm{P}_{\max }\right)$ & up to 200 bar \\
\hline Piston bowl & $\mathrm{d}_{\mathrm{b}}=52 \mathrm{~mm}, 2.2 \mathrm{~mm}$ depth (top hat) \\
\hline Piston optical access & $\mathrm{d}_{\mathrm{w}}=52 \mathrm{~mm}$, quartz window \\
\hline Heating system & $\begin{array}{c}\text { cylinder head and cylinder } \\
\text { liner heating }\end{array}$ \\
\hline Ignition & $\begin{array}{c}\text { spark plug mounted inside } \\
\text { the pre-chamber }\end{array}$ \\
\hline
\end{tabular}

\subsection{LES model}

OpenFOAM v4.x is adopted to solve the LES equations, which are implicitly filtered using the filter $\Delta=\mathrm{V}_{\mathrm{LES}}^{1 / 3}$. For turbulence the k-equation model [24] is used with OpenFOAM default constants. The measured piston position has been imposed in the simulation to ensure the correct compression stroke. Preliminary simulations of single realizations (with identical injection profile) have been performed with three different meshes with a homogeneous cell size within the pre-chamber of $0.125 \mathrm{~mm}, 0.100 \mathrm{~mm}$ and 0.080 $\mathrm{mm}$, leading to approximately 1, 2 and 4 million cells within the pre-chamber. The choice of the LES minimum grid size has been assessed by comparing the temporal evolution of mean and rms of equivalence ratio and turbulence intensity within the pre-chamber for a single compression stroke (not shown here). Simulations with the finer mesh were observed to provide slightly more small flow structures, however the overall flow patterns were found to be very similar between the cases. For this reason, multiple realizations are conducted with a $0.125 \mathrm{~mm}$ cell size.

\section{Example of fuel distribution during injection}

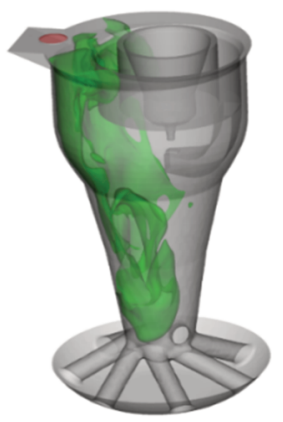

Pre-chamber control volume

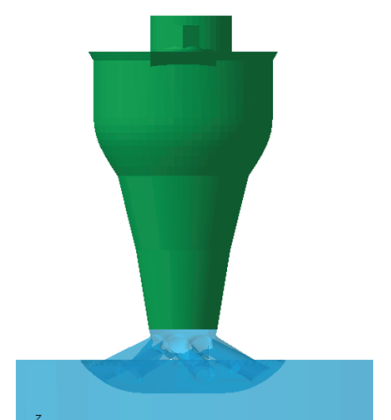

Spark region control volume

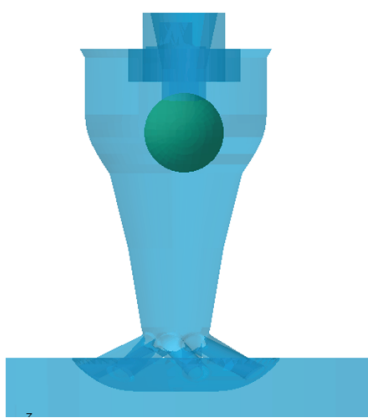

Fig. 2. Left: Example of fuel distribution from LES during the injection event. Centre: Definition of pre-chamber control volume drawn in green. Right: Definition of spark-plug region is a sphere with a $3 \mathrm{~mm}$ radius 


\subsection{RANS model}

RANS simulations have been carried out with the commercial 3D-CFD solver Ricardo VECTIS [20]. RANS equations are solved using the finite volume method. The effects of turbulence are solved by means of Reynolds Averaged Navier-Stokes (RANS) methods, specifically the $\mathrm{k}-\varepsilon$ model time-scale bounded (TSB) model after [12]. As a baseline mesh, a homogeneous grid size of $0.18 \mathrm{~mm}$ has been used within the entire pre-chamber. The RANS grid size has been selected based on a convergence study with respect to the temporal evolution of fuel concentration and turbulence intensity within the pre-chamber. RANS simulations have been carried out with three meshes - referred as Coarse, Medium and Fine - with reference sizes of 0.180, 0.240 and $0.300 \mathrm{~mm}$.

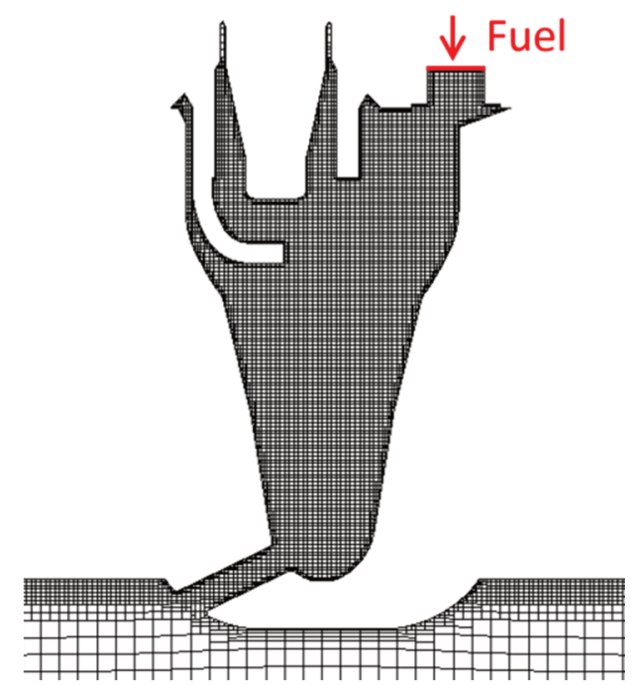

Fig. 3. RANS grid of $0.18 \mathrm{~mm}$ in the spark-plug symmetry plane

\subsection{Fuel injection rate}

Figure 4 shows the profiles of fuel injection rate into the pre-chamber employed for the RANS and LES simulations. In RANS a single profile is used whereas for LES 20 different injection rate profiles have been used to introduce

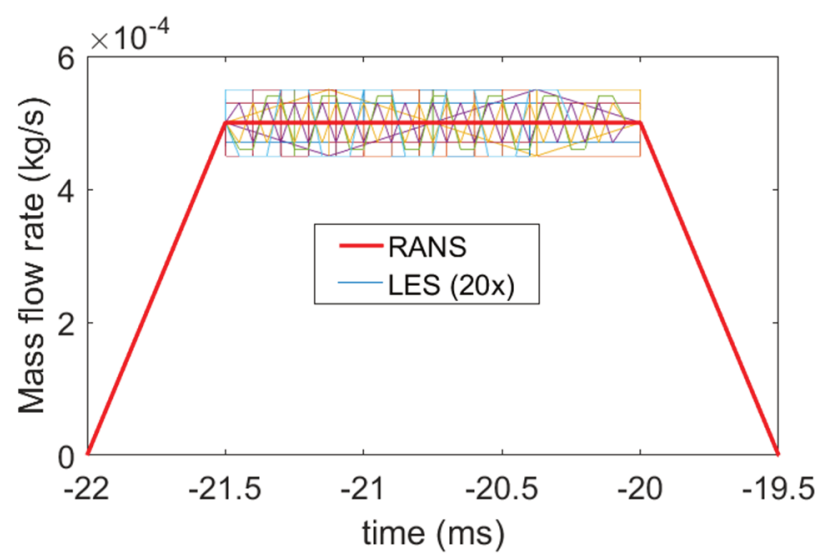

Fig. 4. Profile of fuel injection rate into the pre-chamber used in CFD. Total injected fuel is $1 \mathrm{mg}$. Single RANS profile in red and 20 different profiles for LES a certain degree of perturbation, which as a result will slightly vary the turbulent flow field during the injection event. The total amount of fuel injected has been kept fixed at $1 \mathrm{mg}$, and the effective injection duration is $2.5 \mathrm{~ms}$. The quantity of fuel injection corresponds to an on-average stoichiometric mixture within the pre-chamber at spark time for the test case considered and was found to provide a robust ignition source for the combustion in the main chamber during the experiments.

\section{Results}

Figure 5 displays the temporal evolution of mean and variance of Lambda within the pre-chamber volume (top row) and within the $3 \mathrm{~mm}$ radius sphere around the sparkplug (lower row). The time is referred as time after top dead centre. The nominal spark time is $-3 \mathrm{~ms}$ and is demarked with the red vertical line, whereas the blue vertical line at $22 \mathrm{~ms}$ represents the start of fuel injection into the prechamber. RANS results are shown for the three meshes (Coarse, Medium and Fine) and are compared with the LES mean (red line) arising from averaging the 20 LES realizations.

The mean value of lambda within the pre-chamber (Fig. 5 upper left) before the start of injection $(-22 \mathrm{~ms})$ into the pre-chamber is 2 and corresponds to the main chamber background lambda. During the injection the value of lambda is reduced drastically and shows a minimal value of around 0.3 at the end of injection. Later lambda shows an almost linear increase until top dead centre as a result of the piston motion that induces a flow of lean mixture from the main chamber into the pre-chamber. During the expansion stroke lambda is decreased again because of the fuel stratification in the pre-chamber, where the lean mixture present in the lower half of the pre-chamber leaves first the prechamber and this decreases the mean value of lambda. The comparison between RANS and LES is in good agreement and the same behaviour has been observed with all grid sizes.

The variance of lambda depicted in Fig. 5 upper right shows a different evolution. Before the start of injection, the variance is zero because lambda is homogeneous everywhere. During the injection there is a strong increase of the variance in accordance with the large fuel stratification. During the second half of the injection event the variance decreases because the injected fuel starts to be distributed in the entire pre-chamber. Later the variance increases again because the lean mixture entering through the orifices tends to remain the in lower part of the pre-chamber and it takes some time to mix with the richer mixture arising from the injection. The mixing rate and the lean mixture flow rate from the main chamber are counteracting effects influencing the degree of fuel stratification as quantified by the variance of lambda. At around $-10 \mathrm{~ms}$ the variance of lambda shows a peak and at this point the effect of the two aforementioned phenomena are counterbalanced. During the second half of the compression (between -10 and $0 \mathrm{~ms}$ ) the variance decreases prevalently due to mixing. The comparison between the models shows a reasonable agreement where all cases reproduce the same behaviour. 

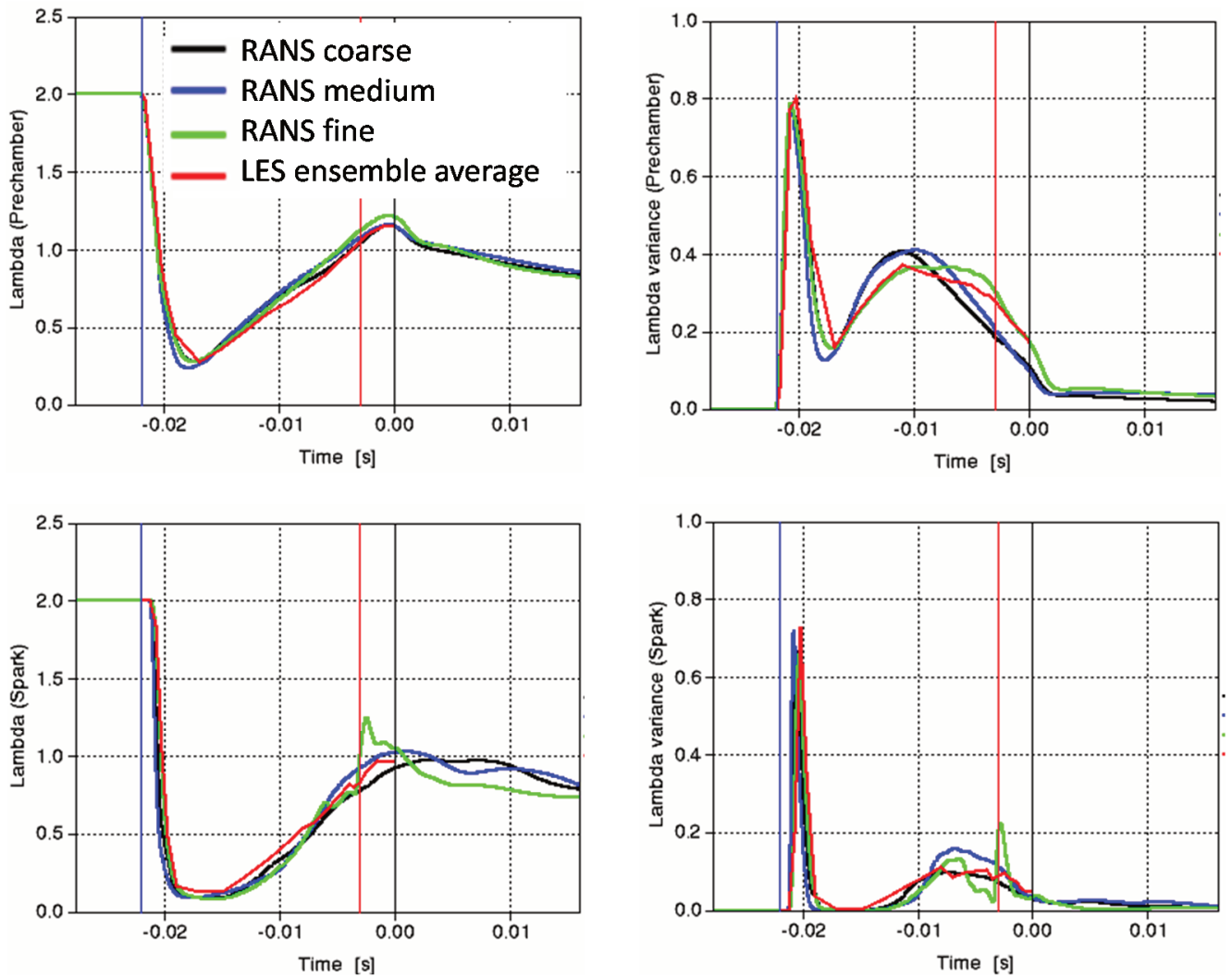

Fig. 5. Temporal evolution of mean and variance of Lambda in the pre-chamber (top row) and in the spark region (lower row). Coarse, Medium and Fine are the RANS grids and red is the LES ensemble average
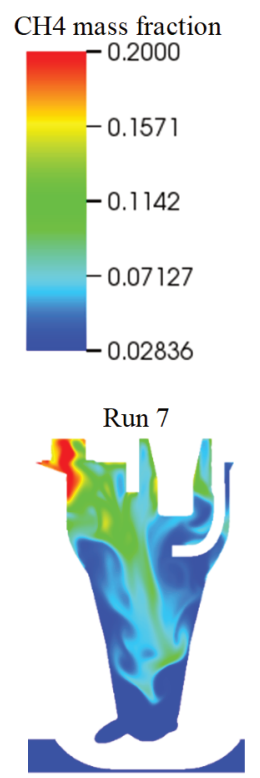

Run 14

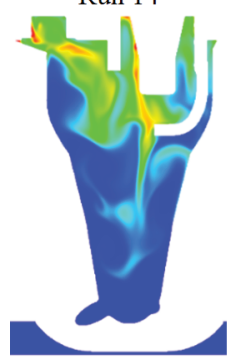

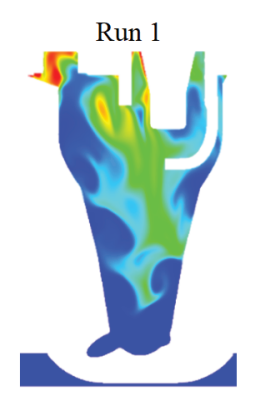

Run 8

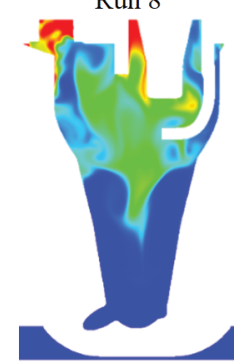

Run 15

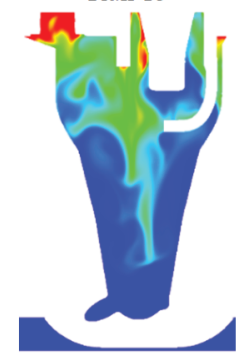

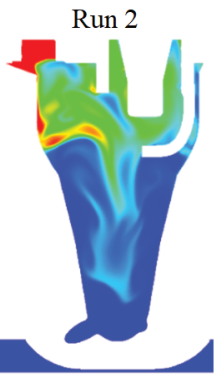
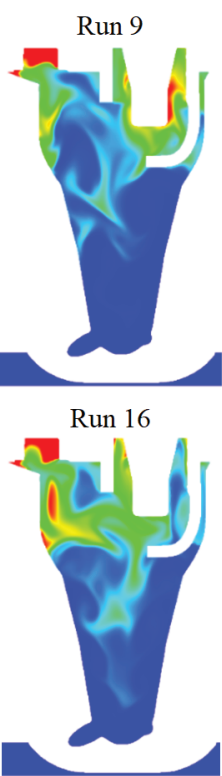

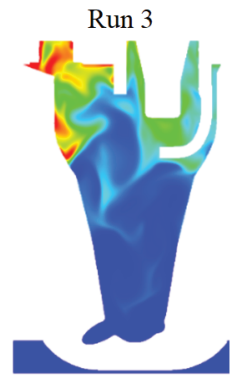

Run 10

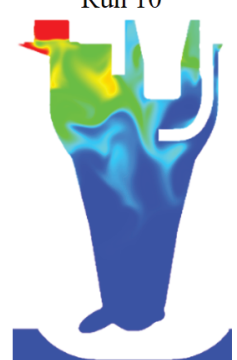

Run 17

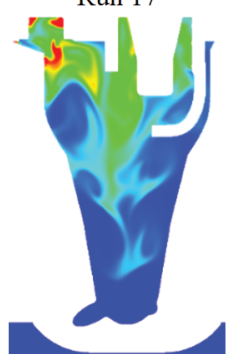

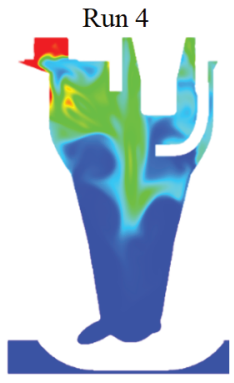

Run 11

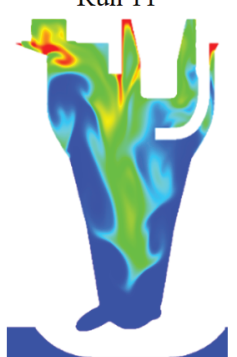

Run 18

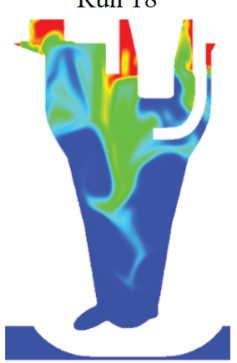

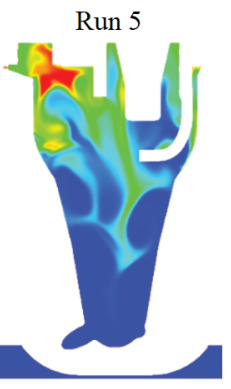

Run 12

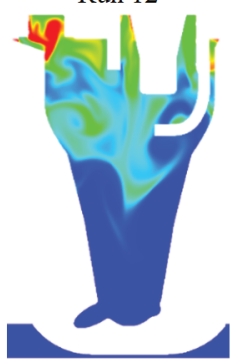

Run 19

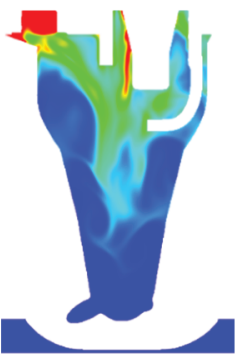

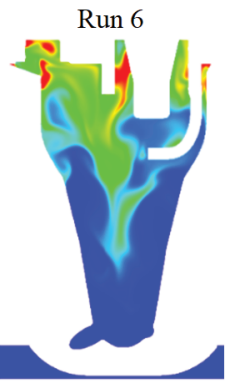

Run 13

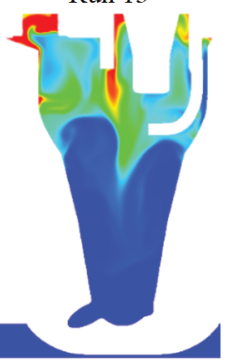

Run 20

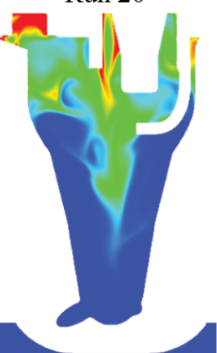

Fig. 6. Fuel mass fraction within the pre-chamber at spark time from 20 independent LES simulation realizations 
$\mathrm{CH}_{4}$ mass fraction (-)

(average 20 runs)

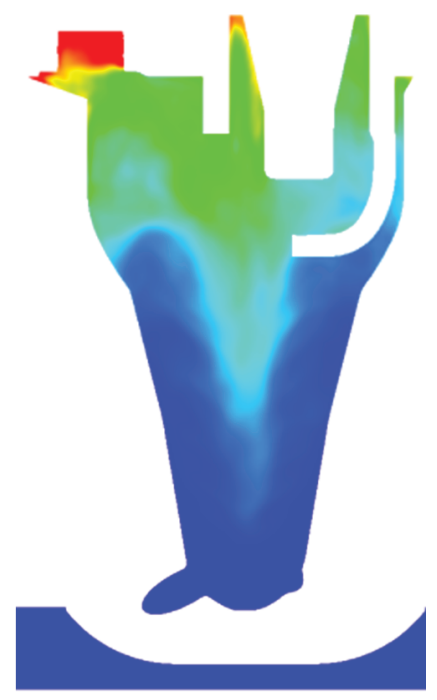

Velocity magnitude $(\mathrm{m} / \mathrm{s})$

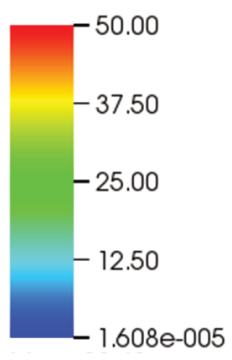

Turbulent kinetic energy $\left(\mathrm{m}^{2} / \mathrm{s}^{2}\right)$
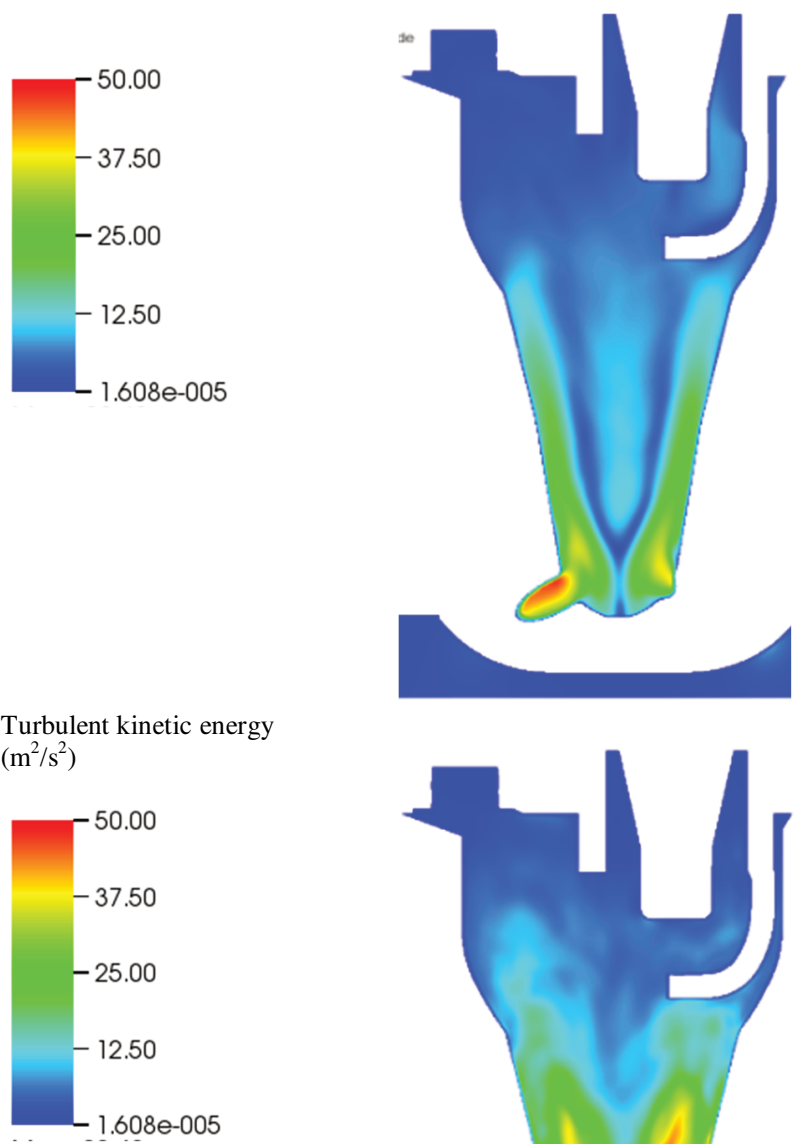

LES

(average 10 runs)
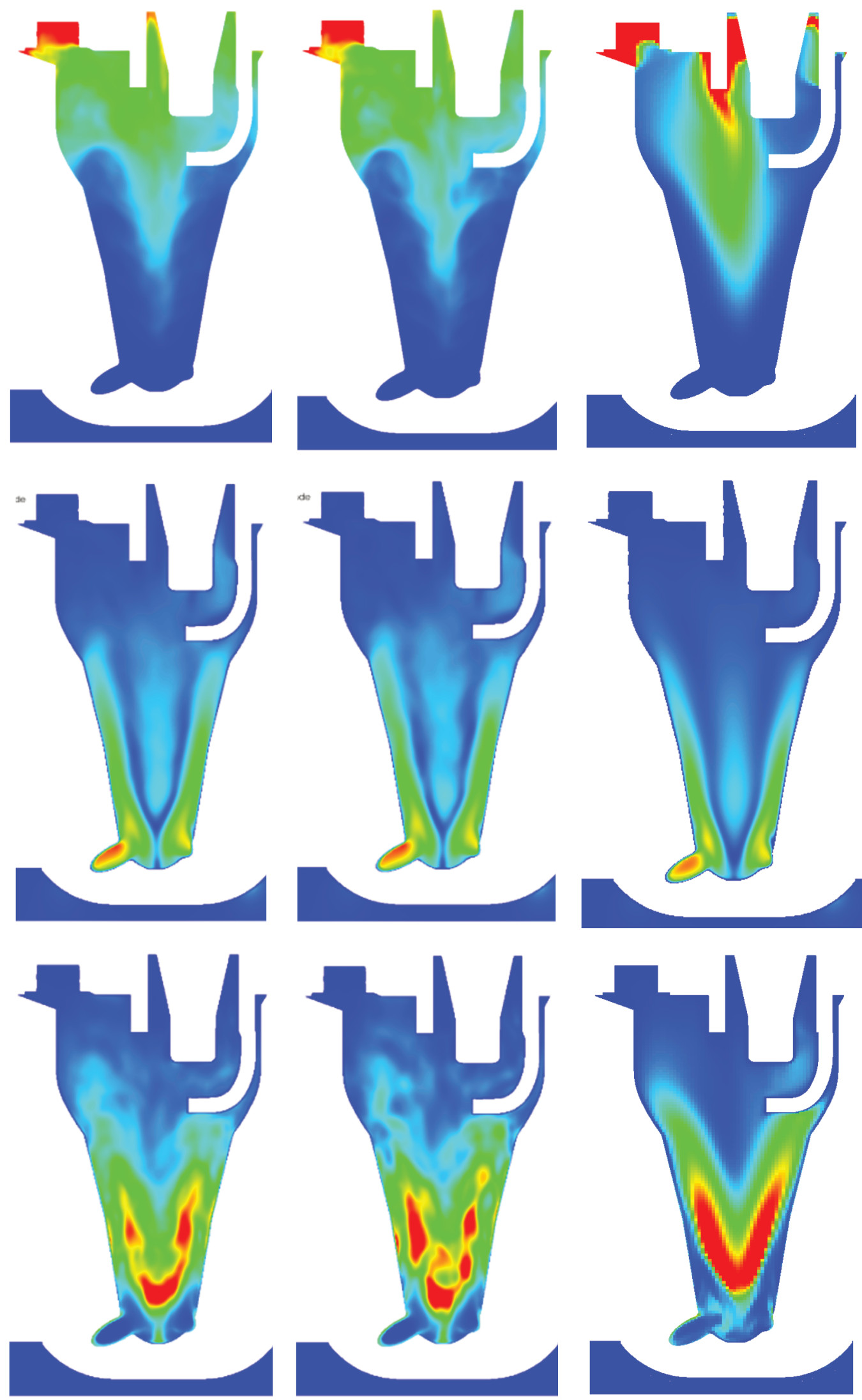

Fig. 7. Spatial distribution of fuel mass fraction (upper), velocity magnitude (middle) and turbulent kinetic energy (lower) at spark time ( 3 ms). LES results have been averaged using 20 (left) and 10 (centre) runs and are compared with RANS (right) 
The same analysis has been performed considering the region close to the spark-plug. This investigation was motivated by the fact that the early flame growth strongly depends on the local conditions around the spark-plug and this has an influence on the overall combustion behaviour and engine performance accordingly. It is important to note that the spark-plug control volume is much smaller than the one embedding the entire pre-chamber and therefore there it contains a considerably smaller number of cells. This is reflected in a noisier and spiky behaviour of the various lines since for some quantities the samples are not enough to guarantee a converged statistic. Nonetheless this doesn't prevent a qualitative assessment of the numerical methods employed. Overall, similar features as for the pre-chamber control volume are observed here, showing good performance of the RANS simulations.

Figure 6 portrays the fuel mass fraction distribution at spark time for the 20 single LES realizations, demonstrating the variability of the flow structures between runs. Note that the same colour scale has been applied for every subfigure. As expected, high fuel concentrations are encountered in the upper half of the pre-chamber because during the compression stroke the lean mixture from the main chamber enters the pre-chamber via the connecting orifices. The tangential orientation of these orifices generates a swirling flow and the fuel rich mixture is trapped in the centre of this helical vortex. Another observation is that there is a high fuel concentration at the deck of the prechamber that has not mixed completely. There are common features between the runs such as a vertical structure of fuel, but the location and concentration vary considerably between realizations. In the lower part of the pre-chamber the fuel concentration corresponds to the background lambda due to the filling process of the pre-chamber. Around the spark-plug there is some degree of variability and on average a richer mixture is present towards the radial centre of the pre-chamber. This means that the early flame generated from the spark is expected to propagate towards the centre and then downwards.

In order to perform a consistent comparison between LES and RANS, the solutions of the 20 LES simulations at spark time have been averaged as illustrated in Fig. 7. Three different quantities have been selected for this com- parison, namely the fuel mass fraction (upper row), the velocity magnitude (middle row) and the turbulent kinetic energy (lower row). In order to understand how sensitive the statistical convergence of the LES results is, two different amounts of LES simulations have been employed to average the results (20 and 10 runs).

The number of runs taken to construct the average shows a weak dependence for the mean fuel mass fraction and velocity magnitude, whereas larger differences are observed for TKE because this is a quantity based on fluctuations of velocity and therefore needs more samples to achieve converged statistics. Nonetheless both with 10 and 20 samples the qualitative distribution of TKE is consistent, and with a larger number of realizations (e.g. 100) no major differences are expected, but rather a smoother shape as seen for the RANS solution.

The RANS results show a very good degree of agreement in terms of velocity and turbulence intensity as well as fuel concentration. This is a promising sign when applying the RANS model in the full engine setup because it builds some confidence on the appropriateness of the turbulence and fuel distribution within the pre-chamber. Based on this comparison the performance of the RANS model is considered as good.

\section{Conclusions}

This paper presents a numerical analysis of fuel concentration and turbulence distribution at spark time for an automotive-sized scavenged pre-chamber mounted at the head of a RCEM by means of RANS and LES turbulence models. The RANS model performance is assessed by a consistent comparison of RANS results with multi-cycle averaged LES results, for which a perturbation of the temporal injection profile of fuel into the pre-chamber has been introduced. Overall, RANS results show that the distributions of the turbulent kinetic energy and fuel concentration at spark-time agree well with the LES ensemble-averaged counterparts. This constitutes a prerequisite in view of the combustion phase and the accuracy reported provides further confidence in this regard.

\section{Acknowledgements}

This work has been supported by the Horizon 2020 GASON project, Grant agreement number 652816.

\section{Bibliography}

[1] ALLISON, P. et al. Pre-chamber ignition mechanism: experiments and simulations on turbulent jet flame structure. Fuel. 2018, 230, 274-281.

[2] BISWAS, S., QIAO, L. A numerical investigation of ignition of ultra-lean premixed $\mathrm{H}_{2}$ /air mixtures by pre-chamber supersonic hot jet. SAE International Journal of Engines. 2017. 2017-01-9284, 2231-2247.

[3] BOLLA, M. et al. Numerical simulations of pre-chamber combustion in an optically accessible RCEM. SAE Technical Paper 2019-01-0224. 2019.

[4] BOLLA, M. et al. Numerical study of turbulence and fuelair mixing within a scavenged pre-chamber using RANS and LES. SAE Technical Paper 2019-01-0198, 2019.

[5] CHINNATHAMBI, P., BUNCE, M., CRUFF, L. RANS based multidimensional modeling of an ultra-lean burn pre- chamber combustion system with auxiliary liquid gasoline injection. SAE Technical Paper 2015-01-0386. 2015.

[6] DALE, J.D., CHECKEL, M.D., SMY, P.R. Application of high energy ignition systems to engines. Progress in Energy and Combustion Science. 1997, 23(5-6), 379-398.

[7] GHOLAMISHEERI, M., GIVLER, S., TOULSON, E. Large eddy simulation of a homogeneously charged turbulent jet ignition system. International Journal of Engine Research. 2017, 1468087417742834.

[8] GHOLAMISHEERI, M., WICHMAN, I.S., TOULSON, E. A study of the turbulent jet flow field in a methane fueled turbulent jet ignition (TJI) system. Combustion and Flame. 2017, 183, 194-206.

[9] HERNÁNDEZ, I., et. al., Flame-wall interaction modelling for pre-chamber combustion in lean burn gas engines, Pro- 
ceedings of 34th International CAE Conference and Exhibition. 2018, Vicenza, Italy.

[10] KOTZAGIANNI, M. et al. Experimental and computational investigations of prechamber jet ignition in a rapid compression expansion machine. Tenth Mediterranean Combustion Symposium. 2017.

[11] LUCAS, G., TALLU, G., WEIßNER, M., CFD-based development of an ignition chamber for a lean and highly efficient CNG combustion, Proc. THIESEL 2018 Conference on Thermo- and Fluid Dynamic Processes in Direct Injection Engines. 2018.

[12] PRZULJ, V. et al. The time scale bounded k- $\varepsilon$ turbulence model and its assessment for automotive applications. In ICHMT digital library online. Begel House Inc. 2012.

[13] SHAPIRO, E. et al. Advanced ignition modelling for prechamber combustion in lean burn gas engines. Proc. 4th International Conference on Ignition Systems for Gasoline Engines. 2018, Berlin.

[14] SHAPIRO, E. et al. Experimental and numerical analysis of pre-chamber combustion systems for lean burn gas engines. SAE Technical Paper 2019-01-0260. 2019.

[15] TALLU, G. et al. 3D CFD modelling and simulation of spark ignition inclusive of turbulence effects and detailed chemical kinetics. Proc. 3rd International Conference on Ignition Systems for Gasoline Engines. 2016, Berlin.

\footnotetext{
Michele Bolla - ETH Zurich, Switzerland.

e-mail:MBolla@lav.mavt.ethz.ch
}

Maria Kotzagianni - ETH Zurich, Switzerland.

e-mail: Kotzagianni@lav.mavt.ethz.ch

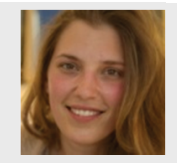

Nick Tiney - Ricardo, Japan.

e-mail:Nick.Tiney@ricardo.com
[16] THELEN, B.C., TOULSON E. A computational study of the effects of spark location on the performance of a turbulent jet ignition system. SAE Technical Paper. 2016.

[17] TOULSON, E., SCHOCK, H.J., ATTARD, W.P. A review of pre-chamber initiated jet ignition combustion systems. 2010, SAE International.

[18] VALIDI, A., SCHOCK, H., JABERI, F. Turbulent jet ignition assisted combustion in a rapid compression machine. Combustion and Flame. 2017, 186, 65-82.

[19] VAVRA, J. et al. Development of a pre-chamber ignition system for light duty truck engine. SAE Technical Paper. 2018.

[20] VECTIS CFD Release 2017.1 Theory Manual. December 2017.

[21] WANG, M. et al. A numerical study on the effects of the orifice geometry between pre-and main chamber for a natural gas engine. SAE Technical Paper. 2017.

[22] WANG, N. et al. The effect of in-cylinder temperature on the ignition initiation location of a pre-chamber generated hot turbulent jet. SAE Technical Paper. 2018.

[23] XU, G. et al. Characterization of combustion in a gas engine ignited using a small un-scavenged pre-chamber. International Journal of Engine Research. 2018, p. 1468087418798918.

[24] YOSHIZAWA, A. Statistical theory for compressible turbulent shear flows, with the application to subgrid modeling. The Physics of Fluids. 1986, 29(7), 2152-2164.

Evgeniy Shapiro - Ricardo, UK.

e-mail: Evgeniy.Shapiro@ricardo.com

Panagiotis Kyrtatos - ETH Zurich \& Vir2sense $\mathrm{GmbH}$.

e-mail:Kyrtatos@lav.mavt.ethz.ch

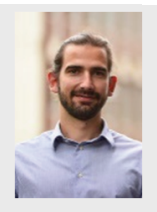

Konstantinos Boulouchos - ETH Zurich, Switzerland.

e-mail:Boulouchos@lav.mavt.ethz.ch 